[Frontiers in Bioscience 4, d816-823, December 1, 1999]

\title{
CELL CYCLE REGULATION OF DNA REPLICATION INITIATION PROTEINS IN MAMMALIAN CELLS
}

\author{
Masatoshi Fujita
}

Masatoshi Fujita, Laboratory of Viral Oncology, Research Institute, Aichi Cancer Center, Kanokoden 1-1, Chikusa-ku, Nagoya 464-8681, Japan

\section{TABLE OF CONTENTS}

\author{
1. Abstract \\ 2. Introduction \\ 3. ORC (Origin recognition complex) \\ 3.1. Budding yeast \\ 3.2. Early embryonic system \\ 3.3. Mammalian somatic cells \\ 4. $C D C 6$ \\ 4.1. Budding yeast \\ 4.2. Early embryonic system \\ 4.3. Mammalian somatic cells \\ 5. MCM (MiniChromosome Maintenance) \\ 5.1. Budding yeast \\ 5.1.1. MCM in pre-RC formation \\ 5.1.2. Complex formation by MCM \\ 5.2. Early embryonic system \\ 5.2.1. Complex formation \\ 5.2.2. Regulation by protein kinases \\ 5.3. Mammalian somatic cells \\ 5.3.1. Chromatin association \\ 5.3.2. Complex formation \\ 5.3.3. Regulation by phosphorylation \\ 6. A suggested hypothetical model for prereplication chromatin and cell cycle regulation in mammalian cells \\ 7. Perspective \\ 8. Acknowledgments \\ 9. References
}

\section{ABSTRACT}

Genomic DNA has to be replicated completely and only once during a single cell cycle in order to maintain integrity. Eukaryotes have developed highly regulated machinery for precisely replicating genomic DNA that is fragmented into multiple chromosomes. Our knowledge of such mechanisms largely depends on findings with budding yeast, since identification of specific DNA sequences acting as replication origins, autonomously replicating sequences, has allowed extensive analyses of the initiation of DNA replication. Several factors essential for regulation of initiation have been identified, including ORC, CDC6 and MCM. Subsequent work has suggested that the fundamental machinery for DNA replication may be conserved in metazoan embryonic cells in which replication occurs sequenceindependently, and also in mammalian nonembryonic cells, where replication origins are more specific. However, there are specific differences. In this review, information on function and regulation of mammalian initiation factors, ORC, CDC6 and MCM, is summarized, and yeast and embryonic systems are compared. A hypothetical model for the state of prereplication chromatin in mammalian cell nuclei and regulation during the cell cycle is also proposed.

\section{INTRODUCTION}

In eukaryotic cells, genomic DNA is fragmented into multiple chromosomes and replication initiates from

multiple origins on these chromosomes. Therefore, the amount of DNA replicated from each origin is relatively little. Thus in metazoans, there is an efficient replication of large amounts of DNA required to expand genome size during evolution. If origin specificity is low and multiple origins fire synchronously, then genome replication is completed rapidly. This is indeed the case in early embryonic cells in metazoans (1). In contrast, in nonembryonic cells, the origins become more specific and firing is highly asynchronous, leading to an increase in S phase length (1). This firing of multiple replication origins throughout $\mathrm{S}$ phase gives rise to at least one problem; since each origin must fire once and only once during a single cell cycle, the already replicated DNA must be clearly differentiated from unreplicated DNA.

Our knowledge of mechanisms responsible for highly regulated eukaryotic DNA replication largely depends on findings with budding yeast, since identification of specific DNA sequences acting as replication origins, autonomously replicating sequences (ARSs), has made way for extensive analysis of the initiation step. Several factors essential for regulation of initiation have been identified, ORC, CDC6 and MCM being representative crucial proteins. An in vitro DNA replication system with Xenopus egg extracts is often used as a model system for metazoan DNA replication. This 
system may mimic the condition in early embryonic cells, where DNA replication occurs sequence-nonspecifically, in contrast to the sequence-specific initiation in budding yeast (1). Nevertheless, Xenopus homologues of yeast initiation factors have also been identified, and shown to be necessary for DNA replication.

Conservation of essential machinery between yeast and embryonic cells suggests that similar events function in DNA replication in mammalian non-embryonic cells. Mammalian homologues of initiation proteins have been identified and appear to be essential for DNA replication. I will summarize here information on function and regulation of initiation factors ORC, CDC6 and MCM in mammalian cells, with a comparison with yeast and embryonic systems.

\section{ORC (ㅁrigin $\underline{\text { Recognition }}$ Complex)}

\subsection{Budding yeast}

ORC, consisting of six subunits ORC1 to 6, was initially identified as binding specifically to ARSs in vitro (2). Findings in genomic footprint analyses $(3,4)$ and immunoprecipitation assays of cross-linked chromatin (5, 6) suggested that ORC binds to ARSs in vivo throughout the cell cycle. Current models suggest that ORC acts as a flag for assembly of other replication proteins such as CDC6 and MCMs, by which the prereplication complex (pre-RC) is formed that is competent for initiation of DNA replication (7).

\subsection{Early embryonic system}

ORC-like complexes probably containing all six ORC subunits have been isolated from Xenopus egg extracts and early Drosophila embryos (8-10). Immunodepletion experiments indicate that XORC (Xenopus ORC) is essential for chromatin binding of XCDC6 and subsequent DNA replication $(8,9,11)$. However, contrary to the situation in the yeast, physical interactions between XORC and XCDC6 have not been evidenced $(9,11)$.

Because DNA replication is sequenceindependent in Xenopus egg extracts (12), XORC may be a low sequence-specific DNA-binding protein. Immunolocalization suggests that it binds to chromatin in interphase $(8,11)$. Biochemical analyses have indicated that XORC2 and XCDC6 are excluded not only from chromatin but also from the nuclear matrix during mitosis (11). However, it was also reported that Drosophila ORC2 remains binding to chromosomes in the mitotic phase (13).

\subsection{Mammalian somatic cells}

Conservation of ORC between yeast and metazoan embryos suggests the existence of an ORC in nonembryonic mammalian cells. However, the properties of mammalian ORC appear somewhat different from those of the embryonic counterpart. Although several human ORC subunits have been identified and physical interactions among them do occur (14-16), complexes apparently containing all six ORC subunits have not been identified. This might simply represent instability of the putative mammalian ORC. However, it has also been suggested that the major, if not the entire, localization in nuclei differs between human ORC1 and 2 . Immunostaining of transfected ORC1 shows nuclear foci, the pattern often representing nuclear matrix localization (17). Biochemical analysis also demonstrated that most endogenous $\mathrm{ORC} 1$ is associated with the matrix throughout the cell cycle (Y. Tatsumi and C. Obuse et al., manuscript in preparation). In contrast, ORC2 is released by nuclease treatment, suggesting chromatin binding $(18,19)$. These findings suggest the existence of ORC subunits that are not associated with the ORC hexamer. One possibility is that some mammalian ORC proteins function in areas other than DNA replication, when they are present in the nonhexamer form.

Why is there the difference in properties of ORC and specificity of origins between embryonic and differentiated cells? One simple possibility is that, metazoans have evolved a special embryonic ORC with low sequence-specificity. If this is not the case, the most possible scenario would be as follows. Metazoan ORC is a DNA-binding protein with relatively low sequencespecificity. In early embryos, there is such a large amount of maternal ORC that the specificity is reduced, leading to sequence-independent DNA replication. In differentiated cells, more complicated nuclear structures and functions have developed, for example for transcription, which does not function in early embryos. Although there are many potential ORC binding sites on genomic DNA, such nuclear events make most sites inaccessible to the ORC. Some are occupied by transcription factors, and others are internalized through formation of high-order chromatin structures. Intranuclear localization of ORC is also specific, for example matrix binding, influencing ORCDNA interactions. As a result, DNA replication initiates from apparently specific sites.

\section{CDC6}

\subsection{Budding yeast}

CDC6 is essential for pre-RC footprint and MCM recruitment $(4,20)$. Chromatin immunoprecipitation studies suggest that CDC6 is associated with ARSs in vivo (5), and this may be linked to CDC6 physical interaction with ORC (21). CDC6 has conserved Walker A and B motifs which are essential for ATP binding and hydrolysis, and mutations in these motifs abrogate CDC6 functions regarding pre-RC footprint formation and/or recruitment of MCMs (22). Furthermore, CDC6 as well as ORC1 and 4 are related to RFC, a loader for PCNA, at the amino acid sequence level (22). MCMs do not appear to be tethered to chromatin via ORC and CDC6 (20). Together, ORC and CDC6 assembled on ARSs may load MCM onto prereplication chromatin, probably using ATP.

Inhibition of re-replication may occur as follows: Once pre-RC is activated, probably by $\mathrm{S}$ phase CDK and CDC7 kinase, it is reversed to replication incompetent post$\mathrm{RC}$, which contains ORC but not other replication proteins such as CDC6 and MCMs (7). While the mechanisms underlying replication-coupled breakdown of pre-RC are currently unknown, inappropriate reassembly is prohibited 
by the $\mathrm{S}$ phase and mitotic CDKs (S-CDK and M-CDK) $(5,7,23)$. In fission yeast, Cdc18, a homologue of CDC6, is phosphorylated by the $\mathrm{CDK}$ and thereby rapidly degraded (24). This regulation of Cdc18 appears critical, since overexpression drives cells into re-replication (24, 25). Although CDC6 protein levels also drop as cells enter $\mathrm{S}$ phase in budding yeast, restoration by the enforced expression can only induce CDC6 association with ARSs but not reloading of MCM (5), indicating that CDC6 protein levels are not an essential target of the CDKs. In metazoans, mitotic CDK phosphorylates CDC6 and MCM without altering their levels (26-28). Therefore, reassembly of pre-RC might be inhibited due to topological incompetence of the phosphorylated CDC6 and MCM for functional interactions.

\subsection{Early embryonic system}

Immunodepletion experiments indicate that XCDC6 is essential for DNA replication in egg extracts (11). Also in this system, $\mathrm{S}$ phase and mitotic CDKs appear to inhibit re-loading of the once dissociated XMCM onto postreplication chromatin $(29,30)$. However, levels of XCDC6 are relatively constant during the cell cycle (11). Phosphorylation of XCDC6 has not been reported. Based on results of immunostaining, XCDC6 appears to bind to chromatin in early interphase, but binds to the nuclear envelope in postreplication nuclei (11).

\subsection{Mammalian somatic cells}

Microinjection of antibodies against mammalian CDC6 inhibits S phase entry (31), suggesting an essential role in DNA replication. CDC6 may be an MCM-loader even in mammalian cells.

Data on mammalian CDC6 can be confusing. Based on findings with immunostaining, nuclear localization seems the case in the G1 phase $(19,26,32,33)$. In NIH3T3 and FT210 cells, almost all CDC6 is partitioned into a detergent insoluble fraction and is not released even by nuclease digestion, suggesting nuclear matrix binding $(19,26)$. Levels of bound CDC6 appear unchanged throughout the cell cycle $(19,26)$. On the other hand, in other cell lines including HeLa, CDC6 is detectable in cytoplasm as cells enter S phase $(26,32,33)$. At least with transfected CDC6, this translocation is controlled by CDK2/Cyclin A (32). Biochemical analyses indicate that in HeLa cells, part of CDC6 is soluble while the remainder binds to the matrix, and that the latter level is constant during the cell cycle (26). Therefore, it may be the soluble form that is translocated in S phase. The matrix-bound, but not soluble, CDC6 is hyperphosphorylated in G2/M phase by $\mathrm{CDC} 2$ kinase (26), which suppresses reloading of MCMs (28). At least in certain cell types, mitotic CDC2phosphorylation of CDC6 does not lead to degradation (26). Selective CDC2 phosphorylation of the bound CDC6 and absence of a soluble form in certain cells imply that it may be the bound form that functions in DNA replication.

\section{MCM (MINICHROMOSOME MAINTENANCE)}

\subsection{Budding yeast}

Six MCM proteins, MCM2 to 7 with a related homology, comprise the MCM family. Their highly conserved central region contains an element similar to the Walker A motif. All six are essential for genome replication. Current concepts suggest that MCM loaded onto pre-replication chromatin plays an essential role in DNA replication, when displaced from chromatin and thereafter CDK activity prevents rebinding $(7,34)$.

\subsubsection{MCM in pre-RC formation}

It has been speculated that MCMs are loaded around ARSs to form pre-RC. The abundance of chromatin-bound MCM appears to be several times that of bound ORC or CDC6, which means the possible existence of multiple MCMs per one ARS (20). Chromatin immunoprecipitation assays have shown that chromatin around, but not distant from, ARSs can be cross-linked to MCM by formaldehyde in the G1 phase, and that the crosslinkable chromatin region moves away from ARSs as the $\mathrm{S}$ phase proceeds $(5,6)$. This could be interpreted as indicating that MCMs associate with ARSs and move with replication forks. Another possible interpretation is that, in G1, the chromatin-bound MCMs are spread over the relatively wide region of chromatin while those near ARSs are in a cross-linkable form. MCMs not associated with ARSs may change to such a cross-linkable form as S phase proceeds.

It has been suggested that MCMs may be activated and function through phosphorylation by CDC7 kinase, which is essential for DNA replication. A subset of $\mathrm{MCM}$ is phosphorylated by $\mathrm{CDC} 7$ in vitro and probably in vivo, and certain MCM mutants can overcome CDC7 dysfunction $(35,36)$.

\subsubsection{Complex formation by MCM}

In metazoans, while hexameric complexes consisting of all six MCMs may be their predominant form, some authors also suggest the existence of more heterogeneous complexes with different compositions (34; also see below). However, it is possible that such heterogeneous complexes are generated in vitro since the heterohexamer tends to be destabilized during experimental processes. Although the existence of the heterohexamer has not been noted in budding yeast, it has been purified from fission yeast (37).

Functions of MCMs remain largely unknown. The observation that a certain subcomplex of human MCMs has weak but ATP-dependent helicase activity is of interest (38). However, such activity has not been identified in other systems. In fission yeast, the purified MCM heterohexamer has no detectable ATPase or helicase activity (37).

\subsection{Early embryonic system}

Immunodepletion experiments suggest that all six XMCMs are essential for DNA replication and that their chromatin binding depends on the XORC and XCDC6 (9, 11, 39, 40). Chromatin-bound XMCMs dissociate as replication proceeds $(39,40)$. In the original licensing model, the nuclear membrane was considered to block rebinding of an essential replication factor, i.e. licensing factor, to chromatin, and MCMs were thought to be 
components of the factor. However, constitutive nuclear localization of mammalian MCM (see below) suggests the non-generality of this model, although it could be important in the embryonic situation (34).

\subsubsection{Complex formation}

In egg extracts, anti-XMCM3 antibodies can precipitate all six XMCMs in approximately equal amounts $(39,40)$, and no change in net molecular mass $(\sim 600 \mathrm{kDa})$ of the XMCM complexes was detected when comparing samples from different cell cycle stages (27). The kinetics of chromatin binding and displacement is synchronous among the six XMCMs (40). Together, these data suggest that the heterohexameric XMCM complexes may be the predominant form. However, in purified XMCM fractions, more heterogeneous XMCM complexes, rather than the heterohexamer, were detected (40).

\subsubsection{Regulation by protein kinases}

Chromatin-bound XMCM4 is phosphorylated in early S phase (41). In yeast and mammals, MCM4 appears to be a substrate of CDC7 kinase $(36,42)$. Therefore, it may be that phosphorylation is achieved by CDC7 which activates XMCM, although direct evidence is lacking. S$\mathrm{CDK}$ is also essential for inducing DNA replication in extracts (7), and as described above, it may also negatively regulate XMCM-chromatin interaction. However, XMCM itself might not be a good substrate for the S-CDK (27). M-CDK phosphorylates XMCM4, and treatment of S phase chromatin with exogenously added M-CDK dissociates XMCMs from chromatin (27). However, in vivo, M-CDK may function to suppress re-loading of already dissociated XMCMs.

\subsection{Mammalian somatic cells}

All six MCMs have been identified in mammals. Microinjection of antibodies against human MCM2 or murine MCM3, or treatment with antisense oligonucleotide against human MCM7 inhibit S phase entry (43-45), suggesting their requirement for DNA replication.

\subsubsection{Chromatin association}

When assessed by immunostaining, mammalian MCMs exclusively show nuclear localization throughout the interphase $(44,46-48)$. These MCMs are biochemically separable into two forms, one extractable by nonionic detergent and the other associated with the nuclei and thus resistant to such extraction $(43,46-48)$. Most of the bound form is released by nuclease treatment, indicating chromatin binding $(46,49,50)$. This is in striking contrast to matrix-associated CDC6 and ORC1. Immunostaining of MCM in detergent-extracted G1 nuclei resulted in homogeneous nuclear staining $(44,46,48)$, which is in contrast to the focal staining observed for ORC1. Taken together, the data suggest that MCMs are bound to chromatin over relatively wide areas rather than in specific locations. While the total amount is constant during the cell cycle, the bound MCM gradually dissociates as cells pass through the $S$ phase $(44,46-48)$.

\subsubsection{Complex formation}

In the soluble fraction, heterohexameric complexes consisting of six MCMs are detected throughout the cell cycle $(28,49,51,52)$. The complexes are unstable but there is strong binding of MCM3 to 5, and MCM4, 6 and 7 form another tight subcomplex (44, 49-53). The hexameric complexes tend to break down into such subcomplexes during experimental procedures. Density gradient centrifugation analyses have also suggested the existence of more heterogeneous MCM complexes $(50,53)$, some may exist in vivo but others might be generated in vitro.

A very important issue is composition of the bound form. Immunoprecipitation of chromatin-bound MCMs solubilized by DNase I digestion under relatively physiological salt concentrations suggests that the predominant form is the heterohexamer $(28,49)$. In immunoprecipitated chromatin-bound MCM complexes, no other protein(s) have to date been detected which have a comparable stoichiometry to MCMs (49), suggesting that there is binding to DNA without mediation of other protein(s) $(20,54)$. However, purified MCMs do not show significant DNA binding activity (37 and our unpublished data). Therefore, it could be that MCM hexamers like PCNA trimers clamp DNA. ATP stabilizes MCM complex-chromatin binding in vitro, without hydrolysis (49), and might therefore function as a co-factor for MCMDNA interactions in vivo.

\subsubsection{Regulation by phosphorylation}

The MCM complexes undergo complicated phosphorylation during the cell cycle. Here, I would like to focus on two points.

One is the phosphorylation of chromatin-bound MCM2 and 4 in the S phase (28). This is reminiscent of S phase XMCM4 phosphorylation (41), and likewise it is conceivable that it is achieved by S-CDK and/or CDC7 kinases. Indeed, MCM2 and 4 appear to be substrates of these kinases in vitro (42). However, it is not clear whether this is the case in vivo. Ishimi showed that a MCM complex with a specific composition, namely a hexamer consisting of two molecules each of MCM4, 6 and 7, has weak but ATP-dependent helicase activity which is inhibited by MCM2 binding $(38,55)$. On the other hand, as described above, the predominant chromatin-bound MCM form appears to be the hexamer consisting of all six MCMs. An intriguing possibility is that $\mathrm{S}$ phase phosphorylation of the latter leads to rearrangement to generate the former active hexamers, which function in DNA replication. However, such has not been demonstrated either in vivo or in vitro.

The other point is hyperphosphorylation of MCM2 and 4 in the G2/M phase, when almost all MCMs are dissociated from chromatin (28). CDC2/cyclin B kinase, a regulator of $\mathrm{G} 2 / \mathrm{M}$ progression, phosphorylates MCM2 and 4 in complexes in vitro, and CDC2 inactivation leads to disappearance of the hyperphosphorylated forms in CDC2 temperature-sensitive mutant murine FT210 cells, which means that the hyperphosphorylation is directed by $\mathrm{CDC} 2 /$ cyclin $\mathrm{B}$ in vivo (28). As described above, the matrix-bound CDC6 is also hyperphosphorylated by $\mathrm{CDC} 2 /$ cyclin $\mathrm{B}$ (26). Furthermore, $\mathrm{CDC} 2$ inactivation in FT210 cells increases the level of chromatin-bound MCMs 




(A) 61 phase



(C) phase

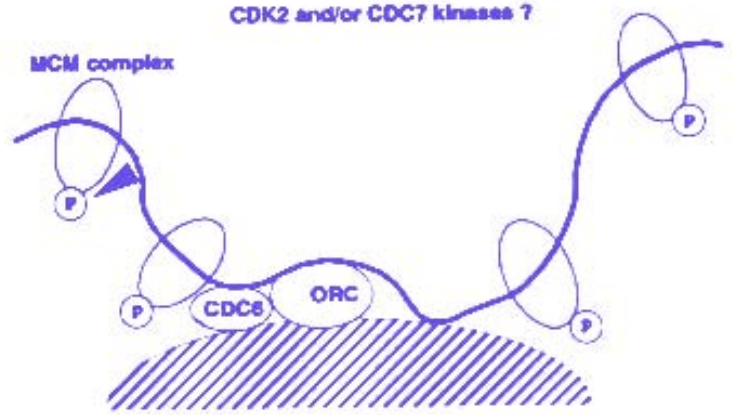

(B) 6 phase

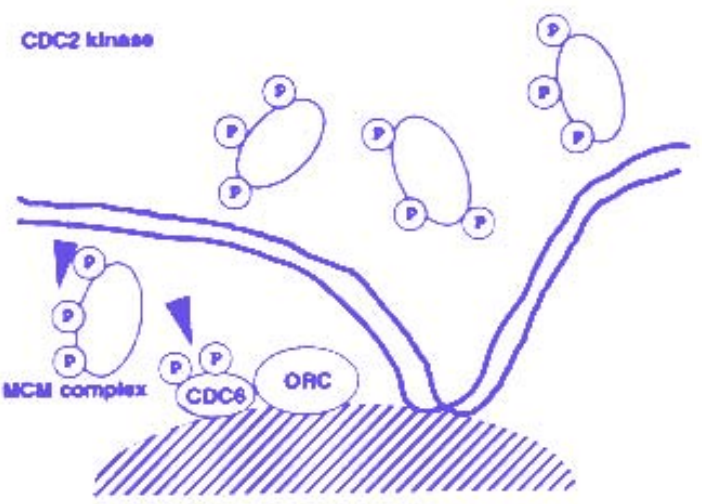

(D) $62 / \mathrm{M}$ phase

Figure 1. A hypothetical model for the state of pre-replication chromatin and cell cycle regulation in mammalian cell. In this model, the soluble CDC6 and MCM present in G1 phase are omitted. Also note that exact phosphorylation sites and numbers in CDC6 and MCM have not been determined, so that the phosphorylation in the model is figurative. (A) In G1 phase, ORC and CDC6 are assumed to be associated with the matrix, MCM complexes being loaded onto chromatin regions not associated with the matrix, including those near ORC/CDC6 binding sites. (B) In $\mathrm{S}$ phase, the bound MCM might be activated through phosphorylation, possibly by CDC7 and/or CDK2 kinases, but this phosphorylation does not induce its dissociation from chromatin. (C) Activated MCM plays an essential role in DNA replication, and is simultaneously displaced from chromatin through an unknown mechanism. In S phase, reloading of once dissociated MCM may be suppressed by CDK2 kinase, possibly through phosphorylation. (D) In G2/M phase, CDC2 kinase hyperphosphorylates CDC6 and MCM complexes, thereby prohibiting reloading of $\mathrm{MCM}$ onto postreplication chromatin. During exit from mitosis, CDC2 kinase activity is restrained and the prereplication chromatin structure is again constructed.

in $\mathrm{G} 2$ phase (28), indicating that mitotic CDK prohibits inappropriate reloading of MCMs onto chromatin and thereby contributes suppression of re-replication, even in mammalian cells. This might occur through the phosphorylation of MCMs and CDC6.

\section{A SUGGESTED HYPOTHETICAL MODEL FOR PREREPLICATION CHROMATIN AND CELL CYCLE REGULATION IN MAMMALIAN CELL}

A hypothetical model for the state of prereplication chromatin and its cell cycle regulation in mammalian cells is shown in figure 1. A putative hexameric mammalian ORC has not been identified, however, given the conservation of ORC in eukaryotes, a mammalian ORC may exist. If so, what is its intranuclear localization? While ORC1 appears to be associated with the matrix, ORC2 binds mainly to chromatin. Expression of MCMs and CDC6, as well as other replication proteins such as PCNA, is cell growth dependent $(31,33,34,47$, 56). Among known mammalian ORC subunits, ORC1, but not ORC2, 4 and 5, appears cell growth dependent $(15,16$, 33). This does not preclude a role for ORCs other than ORC1 in DNA replication, but does imply other function(s). On the other hand, it also suggests that ORC1 may be of prime importance for DNA replication. ORC1 can bind to CDC6 in vitro, and these co-fractionate through multiple column steps from cell lysates (33). In addition, CDC6 is also associated with the matrix (26). Together, in this model, ORC and CDC6 are assumed to be associated 
with the matrix throughout the cell cycle. There are no available data indicating that mammalian ORC/CDC6 binds to chromatin. Again given the conservation of ORC/CDC6 functions, ORC/CDC6 may bind to prereplication chromatin in G1. However, a possible dissociation from chromatin may occur after the $\mathrm{S}$ phase, as suggested for the Xenopus egg system (54).

MCM complexes are loaded, presumably by ORC/CDC6, mainly onto chromatin regions not associated with the matrix. The abundance of chromatin-bound MCM is several times that of bound CDC6 (26), and the relatively large extent of MCM-bound chromatin could explain the pattern of initiation seen in metazoan nonembryonic cells, namely the so-called "initiation zone". Given the analogy with the yeast system, however, it is also possible that at least a small proportion of the complexes is loaded on chromatin near ORC/CDC6 binding sites. The data obtained with immunoprecipitation for lysates prepared from the nuclei chemically cross-linked with Dithiobissuccinimidylpropionate suggest that this is indeed the case (26). The bound MCMs might be activated through phosphorylation possibly by CDC7 and/or CDK2 kinase, but this phosphorylation does not induce dissociation from chromatin $(28,29,54)$. Regarding the role of S-CDK, recently reported findings with yeast and Xenopus eggs that CDC45 protein is loaded onto chromatin, probably via MCM, under the control of S phase $\operatorname{CDK}(57,58)$ are of much interest. The activated MCM plays an essential role in DNA replication, and is simultaneously displaced from chromatin through unknown mechanism(s), coupled to DNA replication. In the $\mathrm{S}$ phase, reloading of once dissociated MCMs may be suppressed by CDK2 kinase (29), possibly through phosphorylation. In the G2/M phase, CDC2 kinase hyperphosphorylates CDC6 and MCM complexes. Reloading the MCM onto postreplication chromatin may thereby be prohibited $(26,28)$. During exit from mitosis, CDC2 kinase activity is restrained and the prereplication chromatin structure is again constructed.

Little is known of the chromatin region associated with these initiation proteins. However, the observation that candidate replication origins in mammals frequently contain matrix attachment region (MAR/SAR) seems indicative (59-61). Chromatin regions associated with ORC1/CDC6 on the matrix might act as centers of initiation zones. Some of the problems with this model could be addressed by chromatin immunoprecipitation assays in mammalian cells.

\section{PERSPECTIVES}

It is important to investigate in detail in vitro biochemical properties of mammalian initiation proteins. Questions of whether or not a mammalian ORC hexamer exists, and if so, what is its binding specificity for DNA, need to be considered. The issue of helicase activity associated with a human MCM complex also warrants further studies. It will be of much importance if an in vitro reconstitution system for ORC-CDC6 binding to DNA and subsequent MCM loading could be established. At the same time, it is also important to ascertain the in vivo situation. For example, actual binding sites of ORC, CDC6 or MCMs in mammalian replicators such as that in the beta-globin locus have not been clarified, and research in this area is expected to provide pertinent information. Functions of the nuclear matrix in DNA replication have long been considered. Understanding the high-order nuclear and chromatin structures is also required. Elucidation of actual processes responsible for DNA replication in mammalian cells will be greatly facilitated by resolution of these issues.

\section{ACKNOWLEDGMENTS}

I thank Dr. C. Obuse (Nara Institute of Science and Technology) for sharing unpublished results, and Dr. T. Tsurumi (Chief, Laboratory of Viral Oncology, Aichi Cancer Center) for critical comment on the manuscript. M. Ohara provided language assistance.

\section{REFERENCES}

1.M.L. DePamphilis: Origins of DNA replication. In: DNA replication in eukaryotic cells. Ed: DePamphilis M.L., Cold Spring Harbor Laboratory Press, NY 45-86 (1996)

2.S.P. Bell \& B. Stillman: ATP-dependent recognition of eukaryotic origins of DNA replication by a multiprotein complex. Nature 357, 128-134 (1992)

3.J.F.X. Diffley, J.H. Cocker, S.J. Dowell \& A.Rowley: Two steps in the assembly of complexes at yeast replication origins in vivo. Cell 78, 303-316 (1994)

4.J.H. Cocker, S. Piatti, C. Santocanale, K. Nasmyth \& J.F.X. Diffley: An essential role for the Cdc6 protein in forming the pre-replicative complexes of budding yeast. Nature 379, 180-182 (1996)

5.T. Tanaka, D. Knapp \& K. Nasmyth: Loading of an Mcm protein onto replication origins is regulated by Cdc6p and CDKs. Cell 90, 649-660 (1997)

6.O.M. Aparicio, D.M. Weinstein \& S.P.Bell: Components and dynamics of DNA replication complexes in $\mathrm{S}$. cerevisiae: redistribution of MCM proteins and Cdc45p during S phase. Cell 91, 59-69 (1997)

7.J.F.X. Diffley: Once and only once upon a time: specifying and regulating origins of DNA replication in eukaryotic cells. Genes Dev 10, 2819-2830 (1996)

8.P.B. Carpenter, P.R. Mueller \& W.G. Dunphy: Role for a Xenopus Orc2-related protein in controlling DNA replication. Nature 379, 357-360 (1996)

9.A. Rowles, J.P.J. Chong, L. Brown, M. Howell, G.I Evan, \& J.J. Blow: Interaction between the origin recognition complex and the replication licensing system in Xenopus. Cell 87, 287-296 (1996)

10.M. Gossen, D.T. Pak, S.K. Hansen, J.K. Acharya \& M.R. Botchan: A Drosophila homolog of the yeast origin recognition complex. Science 270, 1674-1677 (1995)

11.T.R. Coleman, P.B. Carpenter \& W.G. Dunphy: The Xenopus Cdc6 protein is essential for the initiation of a single round of DNA replication in cell-free extracts. Cell 87, 53-63 (1996)

12.J. Walter, L. Sun \& J. Newport: Regulated chromosomal DNA replication in the absence of a nucleus. Mol Cell 1, 519-529 (1998) 
13.D.T.S. Pak, M. Pflumm, I. Chesnokov, D.W. Huang, R. Kellum, J. Marr, P. Romanowski \& M.R. Botchan: Association of the origin recognition complex with heterochromatin and HP-1 in higher eukaryotes. Cell 91, 311323 (1997)

14.K.A. Gavin, M. Hidaka \& B. Stillman: Conserved initiator proteins in eukaryotes. Science 270, 1667-1671 (1995)

15.D.G Quintana, Z. Hou, K.C. Thome, M. Hendricks, P. Saha \& A. Dutta: Identification of $\mathrm{HsORC4}$, a member of the human origin of replication recognition complex. $J$ Biol Chem 272, 28247-28251 (1997)

16.D.G Quintana, K.C. Thome, Z. Hou, A.H. Ligon, C.C. Morton \& A. Dutta: ORC5L, a new member of the human origin recognition complex, is deleted in uterine leiomyomas and malignant myeloid diseases. J Biol Chem 273, 2713727145 (1998)

17.D.A. Wolf, D. Wu \& F. Mckeon: Disruption of rereplication control by overexpression of human ORC1 in fission yeast. $J$ Biol Chem 271, 32503-32506 (1996)

18.M. Ritzi, M. Baack, C. Musahl, P. Romanowski, R.A. Laskey \& R. Knippers: Human minichromosome maintenance protein and human origin recognition complex 2 protein on chromatin. J Biol Chem 273, 24543-24549 (1998)

19.K. Stoeber, A.D. Mills, Y. Kubota, T. Krude, P. Romanowski, K. Marheineke, R.A. Laskey \& G.H. Williams: Cdc6 protein causes premature entry into $\mathrm{S}$ phase in a mammalian cell-free system. EMBOJ 17, 7219-7229 (1998)

20.S. Donovan, J. Harwood, L.S. Drury \& J.F.X. Diffley: Cdc6p-dependent loading of Mcm proteins onto pre-replicative chromatin in budding yeast. Proc Natl Acad Sci USA 94, 5611-5616 (1997)

21.C. Liang, M. Weinreich \& B. Stillman: ORC and Cdc6p interact and determine the frequency of initiation of DNA replication in the genome. Cell 81, 667-676 (1995)

22.G. Perkins \& J.F.X. Diffley: Nucleotide-dependent prereplicative complex assembly by Cdc6p, a homolog of eukaryotic and prokaryotic clamp-loaders. Mol Cell 2, 23-32 (1998)

23.S. Piatti, T. Bohm, J.H. Cocker, J.F.X. Diffley \& K. Nasmyth: Activation of S-phase-promoting CDKs in late G1 defines a "point of no return" after which Cdc6 synthesis cannot promote DNA replication in yeast. Genes Dev 10, 1516-1531 (1996)

24.P.V. Jallepalli, G.W. Brown, M. Muzi-Falconi, D. Tien \& T.J. Kelly: Regulation of the replication initiator protein p65cdc18 by CDK phosphorylation. Genes Dev 11, 2767-2779 (1997)

25.H. Nishitani \& P. Nurse: p65cdc18 plays a major role controlling the initiation of DNA replication in fission yeast. Cell 83, 397-495 (1995)

26.M. Fujita, C. Yamada, H. Goto, N. Yokoyama, K. Kuzushima, M. Inagaki \& T. Tsurumi: Cell Cycle regulation of human CDC6 protein: intracellular localization, interaction with the human MCM complex and CDC2 kinase-mediated hyperphosphorylation. J Biol Chem 274, 25927-25932 (1999)

27.M. Hendrickson, M. Madine, S. Dalton \& J. Gautier Phosphorylation of MCM4 by cdc2 protein kinase inhibits the activity of the minichromosome maintenance complex. Proc Natl Acad Sci USA 93, 12223-12228 (1996)

28.M. Fujita, C. Yamada, T. Tsurumi, F. Hanaoka, K. Matsuzawa \& M. Inagaki: Cell cycle- and chromatin binding state-dependent phosphorylation of human MCM heterohexameric complexes: a role for cdc2 kinase. $J$ Biol Chem 273, 17095-17101 (1998)

29.X.H. Hua, H. Yan \& J. Newport: A role for Cdk2 kinase in negatively regulating DNA replication during $\mathrm{S}$ phase of the cell cycle. J Cell Biol 137, 183-192 (1997)

30.H.M. Mahbubani, J.P.J. Chong, S. Chevalier, P. Thömmes \& J.J. Blow: Cell cycle regulation of the replication licensing system: involvement of a Cdk-dependent inhibitor. J Cell Biol 136, 125-135 (1997)

31.Z. Yan, J. DeGregori, R. Shohet, G. Leone, B. Stillman, J.R. Nevins \& R.S. Williams: Cdc6 is regulated by E2F and is essential for DNA replication in mammalian cells. Proc Natl Acad Sci USA 95, 3603-3608 (1998)

32.B.O. Petersen, J. Lukas, C.S. Sørensen, J. Bartek \& K. Helin: Phosphorylation of mammalian CDC6 by Cyclin A/CDK2 regulates its subcellular localization. EMBO J 18, 396-410 (1999)

33.P. Saha, J. Chen, K.C. Thome, S.J. Lawlis, Z. Hou, M. Hendricks, J.D. Parvin \& A. Dutta: Human CDC6/Cdc18 associates with Orc1 and Cyclin-cdk and is selectively eliminated from the nucleus at the onset of S phase. Mol Cell Biol 18, 2758-2767 (1998)

34.S.E. Kearsey \& K. Labib: MCM proteins: evolution, properties, and role in DNA replication. Biochim Biophys Acta 1398, 113-136 (1998)

35.C.F.J. Hardy, O. Dryga, P.M.B. Pahl \& R.A. Sclafani: $m c m 5 / c d c 46-b o b 1$ bypasses the requirement for the $\mathrm{S}$ phase activator Cdc7p. Proc Natl Acad Sci USA 94, 3151-3155 (1997)

36.M. Lei, Y. Kawasaki, M.R. Young, M. Kihara, A. Sugino \& B.K. Tye: Mcm2 is a target of regulation by Cdc7-Dbf4 during the initiation of DNA synthesis. Genes Dev 11, 33653374 (1997)

37.Y. Adachi, J. Usukura \& M. Yanagida: A globular complex formation by Ndal and the other five members of the MCM protein family in fission yeast. Genes Cells 2, 467-479 (1997)

38.Y. Ishimi: A DNA helicase activity is associated with an MCM4, -6, and -7 protein complex. J Biol Chem 272, 2450824513 (1997)

39.Y. Kubota, S. Mimura, S. Nishimoto, T. Masuda, H. Nojima \& H. Takisawa: Licensing of DNA replication by a multi- protein complex of MCM/P1 proteins in Xenopus eggs. EMBO J 16, 3320-3331 (1997)

40.P. Thömmes, Y. Kubota, H. Takisawa \& J.J. Blow: The RLF-M component of the replication licensing system forms complexes containing all six MCM/P1 polypeptides. EMBO J 16, 3312-3319 (1997)

41.M. Coué, S.E. Kearsey \& M. Méchali: Chromatin binding, nuclear localization and phosphorylation of Xenopus cdc21 are cell-cycle dependent and associated with the control of initiation of DNA replication. EMBO J 15, 1085-1097 (1996) 42.N. Sato, K. Arai \& H. Masai: Human and Xenopus cDNAs encoding budding yeast $\mathrm{Cdc} 7$-related kinases: in vitro phosphorylation of MCM subunits by a putative human homologue of Cdc7. EMBO J 16, 4340-4351 (1997)

43.I.T. Todorov, R. Pepperkok, R. Philipova, S.E. Kearsey, W. Ansorge \& D. Werner: A human nuclear protein with sequence homology to a family of early $\mathrm{S}$ phase proteins is required for entry into $\mathrm{S}$ phase and for cell division. $J$ Cell Science 107, 253-265 (1994)

44.H. Kimura, N. Nozaki \& K. Sugimoto: DNA polymerase alpha associated protein $\mathrm{P} 1$, a murine homolog 
of yeast MCM3, changes its intranuclear distribution during the DNA synthetic period. EMBO J 13, 4311-4320 (1994)

45.M. Fujita, T. Kiyono, Y. Hayashi \& M. Ishibashi: Inhibition of S-phase entry of human fibroblasts by an antisense oligomer against hCDC47. Biochem Biophys Res Commun 219, 604-607 (1996)

46.I.T. Todorov, A. Attaran \& S.E. Kearsey: BM28, a human member of the MCM2-3-5 family, is displaced from chromatin during DNA replication. J Cell Biol 129, 14331445 (1995)

47.M. Fujita, T. Kiyono, Y. Hayashi \& M. Ishibashi: hCDC47, a human member of the MCM family: dissociation of the nucleus-bound form during $\mathrm{S}$ phase. $J$ Biol Chem 271, 4349- 4354 (1996)

48.T. Krude, C. Musahl, R.A. Laskey \& R. Knippers: Human replication protein $\mathrm{hCdc} 21, \mathrm{hCdc} 46$ and $\mathrm{P} 1 \mathrm{Mcm} 3$ bind chromatin uniformly before S-phase and are displaced locally during DNA replication. J Cell Sci 109, 309-318 (1996)

49.M. Fujita, T. Kiyono, Y. Hayashi \& M. Ishibashi: In vivo interaction of human MCM heterohexameric complexes with chromatin: possible involvement of ATP. J Biol Chem 272, 10928-10935 (1997)

50.R. Burkhart, D. Schulte, B. Hu, C. Musahl, F. Göhring \& R. Knippers: Interactions of human nuclear proteins P1Mcm3 and P1Cdc46. Eur $J$ Biochem 228, 431-438 (1995)

51.A. Richter \& R. Knippers: High-molecular-mass complexes of human minichromosome-maintenance proteins in mitotic cells. Eur J Biochem 247, 136-41 (1997) 52.H. Kimura, T. Ohtomo, M. Yamaguchi, A. Ishii \& K. Sugimoto: Mouse MCM proteins: complex formation and transportion to the nucleus. Genes Cells 1, 977-993 (1996) 53.C. Musahl, D. Schulte, R. Burkhart \& R. Knippers: A human homologue of the yeast replication protein $\mathrm{Cdc} 21$ : interaction with other Mcm proteins. Eur J Biochem 230, 1096-1101 (1995)

54.X.H. Hua \& J. Newport: Identification of a preinitiation step in DNA replication that is independent of origin recognition complex and cdc6, but dependent on $\operatorname{cdk} 2$. $J$ Cell Biol 140, 271-281 (1998)

55.Y. Ishimi, Y. Komamura, Z. You \& H. Kimura: Biochemical function of mouse minichromosome maintenance 2 protein. J Biol Chem 273, 8369-8375 (1998) 56.K. Ohtani, A. Tsujimoto, M. Ikeda \& M. Nakamura: Regulation of cell growth-dependent expression of mammalian CDC6 gene by the cell cycle transcription factor E2F. Oncogene 17, 1777-1785 (1998)

57.L. Zou \& B. Stillman: Formation of a preinitiation complex by S-phase cyclin CDK-dependent loading of Cdc45p onto chromatin. Science 280, 593-596 (1998)

58.S. Mimura \& H. Takisawa: Xenopus Cdc45-dependent loading of DNA polymerase alpha onto chromatin under the control of S-phase Cdk. EMBO J 17, 5699-5707 (1998) 59.P.R. Cook: The nucleoskeleton:artefact, passive framework or active site? J Cell Science 90, 1-6 (1988)

60.P.A. Dijkwel, J.P Vaughn \& J.L. Hamlin: Mapping of replication initiation sites in mammalian genomes by twodimensional gel analysis: stabilization and enrichment of replication intermediates by isolation on the nuclear matrix. Mol Cell Biol 11, 3850-3859 (1991)
61.W.R. Walter, G.B. Singh \& S.A. Krawetz: MARs mission update. Biochem Biophys Res Commun 242, 419422 (1998)

Key words: DNA replication, mammalian cell, initiation factor, ORC, CDC6, MCM, CDK, Review

Send correspondence to: Dr Masatoshi Fujita, Laboratory of Viral Oncology, Research Institute, Aichi Cancer Center, Kanokoden 1-1, Chikusa-ku, Nagoya 464-8681, Japan, Tel/Fax: 81-52-764-2981, E-mail: mfujita@aichigw.aichicc.pref.aichi.jp

Received 9/15/99 Accepted 10/15/99 